\title{
On the plane strain in a theory for self-gravitating elastic configuration with initial static stress field $\left({ }^{*}\right)$
}

\author{
E. BoschI $(* *)$
}

Received on March 22nd, 1974

Summary. - This paper is concerned with the plane strain in a theory for an arbitrary, uniformly rotating, self-gravitating, perfectly elastic Earth model with a hydrostatic initial stress ficld. Using the associated matrices method, a representation of Galerkin type is given. This representation enables us to derive the solution of the vibration problem corresponding to concentrated body forces.

Riassunto. --- In questo lavoro si tratta il problema della deformazione piana nell'ambito di un modello terrestre arbitrario, uniformemente ruotante, autogravitante, perfettamente elastico e soggetto ad un campo idrostatico di sforzo iniziale. Usando il metodo delle matrici associate, viene data una rappresentazione di tipo Galerkin. Questa rappresentazione permette la soluzione del problema delle vibrazioni corrispondenti a forze di massa concentrate.

\section{INTRODUCTION.}

Dahlen ( $\left.{ }^{3}\right)$ has developed the linearized equations and linearized boundary and continuity conditions governing small elastic-gravitational disturbances away from equilibrium of an arbitrary uniformly rotating, self-gravitating, perfectly elastic Earth model with an arbitrary initial static stress field [see, also, Boschi (1)].

(*) This work has been made during a tenure of a C.N.R. fellowship. Cavendish Laboratory, University of Cambridge.

(**) On leave from Istituto di Geofisica, Universiti di Bologna, and Istituto di Scienze della Terra, Università di Aneona. 
In this paper we consider this theory in the case of a hydrostatic initial stress field and derive the equations for the plane strain. The medium is assumed homogeneous and isotropic. By making use of the associated matrices methor $\left({ }^{5}\right)$, we give a representation of Galerkin type. This representation enables us to obtain the solution corresponding to concentrated loads in an infinite medium for the case of stationary vibrations. Analogous problems have been studied in other fields. $(6,4,2)$

\section{BASIC EQTATIONS.}

In the following we employ a rectangular coordinate system O: $x_{k}$ and the usual indicial notations. The Greek indices are supposed to take the values 1,2 and the Iuatin indices the values 1, 2, 3 .

Let $\Sigma$ be a plane region occupied by the considered medium.

We denote by $s_{a}$ the components of the displacement vector and by $\Phi_{1}$ the perturbation in the gravitational potential.

In the case of plane strain, we have:

$$
s_{\alpha}=s_{\alpha}\left(x_{1}, x_{2}, t\right) \quad, \quad \Phi_{1}=\Phi_{1}\left(x_{1}, x_{2}, t\right), s_{3}=0 .
$$

From the equations established by Dahlen $\left({ }^{3}\right)$, we can derive the following basic equations for the plane strain problem in the case of a hydrostatic initial stress tensor $T_{i j}^{o}=-p_{\circ} \delta_{i j}\left(p_{0}=\right.$ const.):

- the equations of motion:

$$
\begin{gathered}
\Phi_{1, \alpha \alpha}=-4 \pi \varrho_{0} G s_{\beta, \beta} \\
\varrho_{\circ} \ddot{s}_{1}-2 \varrho_{0} \Omega_{3} \dot{s}_{2}=-\varrho_{0} Z_{1,1}+\tilde{T}_{\beta_{1}, \beta}+H_{1}^{\prime} \\
\varrho_{\circ} \ddot{s}_{2}-2 \varrho_{0} \Omega_{3} \dot{s}_{1}=-\varrho_{0} \Phi_{1,2}+\tilde{T}_{\beta_{2}, \beta}+H_{2}^{\prime}
\end{gathered}
$$

- the constitutive equations:

$$
\begin{aligned}
& \widetilde{T}_{11}=\left(\lambda+2 \mu-2 p_{\mathrm{o}}\right) s_{1,1}+\left(\lambda-p_{\mathrm{o}}\right) s_{2,2} \\
& \widetilde{T}_{22}=\left(\lambda-p_{\mathrm{o}}\right) s_{1,1}+\left(\lambda+2 \mu-2 p_{\mathrm{o}}\right) s_{2,2} \\
& \tilde{T}_{12}=\mu s_{2,1}+\left(\mu-p_{0}\right) s_{1,2} \\
& \tilde{T}_{21}=\mu s_{1,2}+\left(\mu-p_{0}\right) s_{2,1}
\end{aligned}
$$

In the above equations, we have used the following notations: $\widetilde{T}_{\alpha \beta}$ - the components of the incremental pseurlostress tensor; $F_{\alpha}-$ the components of body forces; $\vec{\Omega}=\left(0,0, \Omega_{3}\right)$ - the steady angular 
velocity rotation; $\lambda, \mu$ - the appropriate constants of the material; Qo - the mass density; $G$ - the gravitational constant; a comma denotes partial differentiation with respect to space variables and a superposed dot denotes partial differentiation with respect to the time $t$.

Using equations [4], the differential equations [2] and [3] may be written as:

$$
\begin{gathered}
{\left[\mu \Delta+\left(\lambda+\mu-2 p_{0}\right) \frac{\partial^{2}}{\partial x_{1}^{2}}-\varrho_{\circ} \frac{\partial^{2}}{\partial t^{2}}\right] s_{1}+\left[\left(\lambda+\mu-2 p_{0}\right) \frac{\lambda_{2}}{\partial x_{1} \partial x_{2}}+\right.} \\
\left.+2 \varrho_{0} \Omega_{3} \frac{\partial}{\partial t}\right] s_{2}-\varrho_{0}-\frac{\partial}{\partial x_{1}} \Phi_{1}=-F_{1} \\
{\left[\left(\lambda+\mu-2 p_{\circ}\right) \frac{\partial^{2}}{\partial x_{1} \partial x_{2}}-2 \varrho_{0} \Omega_{3} \frac{\partial}{\partial t}\right] s_{1}+\left[\mu \Delta+\left(\lambda+\mu-2 p_{\circ}\right)\right.} \\
\left.\frac{\partial^{2}}{\partial x_{2}^{2}}-\varrho_{0} \frac{\partial^{2}}{\partial t^{2}}\right] s_{2}-\varrho_{0} \frac{\partial}{\partial x_{2}} \Phi_{1}=-F_{2} \\
4 \pi \varrho_{0} G \frac{\partial}{\partial x_{1}} s_{1}+4 \pi \varrho_{0} G \frac{\partial}{\partial x_{2}} s_{2}+\Delta \Phi_{1}=0
\end{gathered}
$$

\section{GALERKIN REPRESENTATION.}

Using the associated matrices method $\left({ }^{5}\right)$, we obtain the following representation of Galerkin type:

$$
\begin{aligned}
& s_{1}=\mu\left\{\left|\square_{2}^{2} ;-\frac{v_{1}{ }^{2}}{v_{2}{ }^{2}} \frac{\partial^{2}}{\partial x_{2}{ }^{2}}\right| \Delta+\frac{4 \pi \varrho_{0} G}{v_{2}{ }^{2}} \frac{\partial^{2}}{\partial x_{2}{ }^{2}}\right\} \Gamma_{1} \\
& -\left\{\left[\left(\lambda+\mu-2 p_{0}\right) \frac{\partial^{2}}{\partial x_{1} \partial x_{2}}+2 \varrho_{0} \Omega_{3} \frac{\partial}{\partial t}\right] A+4 \pi \varrho_{0}^{2} \theta \frac{u-}{\partial x_{1}} \partial x_{2}\right\} \Gamma_{2} \\
& +\mu \varrho_{0}\left\lceil\square_{2}^{2} \frac{\partial}{\partial x_{1}}-\frac{2 \Omega_{3}}{v_{2}^{2}} \frac{\partial^{2}}{\partial x_{2} \partial t}\right\rceil \Gamma_{3} \text {. } \\
& s_{2}=-\left\{\left[\left(\lambda+\mu-2 p_{0}\right) \frac{\partial^{2}}{\partial x_{1} \partial x_{2}}-2 \varrho_{0} \Omega_{3} \frac{\partial}{\partial t}\right] \Delta+4 \pi \varrho_{0}^{2} G \frac{\partial^{2}}{\partial x_{1} \partial x_{2}}\right\} \Gamma_{1} \\
& +\mu\left\{\left[\Pi_{2}^{2}+\frac{v_{1}^{2}}{v_{2}^{2}} \frac{\partial^{2}}{\partial x_{1}^{2}}\right] \Delta+\frac{1 \approx \approx \approx}{v_{2}^{2}} \frac{\lambda 2}{\partial x_{1}^{2}}\right\} \Gamma_{2} \\
& +\mu \varrho_{0}\left[\square_{2}^{2} \frac{\partial}{\partial x_{0}}+\frac{2 \Omega_{3}}{v_{2}^{2}} \frac{\partial^{2}}{\partial x_{1} \partial t}\right] \Gamma_{3}
\end{aligned}
$$




$$
\begin{aligned}
& \left.\Phi_{1}=-1 \pi \varrho_{0} G \mu \mid \square_{2}^{2} \frac{\partial}{\partial x_{1}}+\frac{2 \Omega_{3}}{v_{2}^{2}} \frac{\partial^{2}}{\partial x_{2} \partial t}\right] \Gamma_{1} \\
& -4 \pi \varrho_{0} G \mu\left|\square_{2}^{2} \frac{\partial}{\partial x_{2}}-\frac{2 \Omega_{3}}{v_{2}^{2}} \frac{\partial^{2}}{\partial x_{1} \partial t}\right| \Gamma_{2} \\
& +\mu\left(\lambda+2 \mu-2 p_{0}\right)\left[\square_{1}^{2} \square_{2}^{2}+\frac{4 \Omega_{3}{ }^{2}}{v_{1}^{2} v_{2}{ }^{2}} \frac{\partial^{2}}{\partial t^{2}}\right] \Gamma_{3}
\end{aligned}
$$

where

$$
\begin{aligned}
& \square_{\alpha}^{2}=\Delta-\frac{1}{v_{\alpha^{2}}} \frac{\partial^{2}}{\partial t^{2}} \quad, \quad \Delta=\frac{\partial^{2}}{\partial x_{\alpha}{ }^{2}} \\
& v_{1}^{2}=\left(\lambda+2 \mu-2 p_{0}\right) / \varrho_{0} \\
& v_{2}^{2}=\mu / \varrho_{0}
\end{aligned}
$$

The functions $\Gamma_{k}=\Gamma_{k}\left(x_{1}, x_{2}, t\right)$ satisfy to the following equations:

$$
\begin{aligned}
& D \Gamma_{\alpha}-\frac{1}{\mu\left(\lambda+2 \mu-2 p_{0}\right)} F_{n} \\
& D \Gamma_{3}=0
\end{aligned}
$$

where

$$
D=\left(\square_{1}^{2} \square_{2}^{2}+\frac{4 \pi \varrho_{0} G}{v_{2}^{2}} \square_{3}^{2}\right) \Delta
$$

where

$$
\square_{3}^{2}=\Delta-\frac{1}{v_{3}{ }^{2}} \frac{\partial^{2}}{\partial t^{2}} \quad ; \quad v_{\underline{2}}^{2}=v_{\underline{2}}^{2}\left(1-\frac{\Omega_{3}^{2}}{\pi \varrho_{0} G}\right)^{-1}
$$

\section{STATIONARY VIBRATIONS.}

In what follows, we assume that:

$$
F_{\alpha}=R_{e}\left\{F_{o}^{*}\left(x_{1}, x_{2}\right) e^{i \omega t}\right\}
$$

In this case we seek the solution in the form:

$$
\begin{aligned}
& s_{\alpha}=R_{e}\left\{s_{\alpha}^{*}\left(x_{1}, x_{2}\right) e^{i \omega t}\right\}, \\
& \Phi_{1}=\operatorname{Re}\left\{\Phi_{1}^{*}\left(x_{1}, x_{2}\right) e^{i \omega t}\right\} .
\end{aligned}
$$


From the Galerkin representation [6], by putting

$$
\Gamma_{j}=R_{e}\left\{\Gamma_{j}^{*}\left(x_{1}, x_{2}\right) e^{i \omega t}\right\}
$$

we obtain

$$
\begin{aligned}
& s_{:}^{\therefore}=\mu\left\{\left[\square_{2}^{* 2}+\frac{v_{1}^{2}}{v_{2}^{2}} \frac{\partial^{2}}{\partial x_{2}{ }^{2}}\right] \Delta+\frac{4 \pi \varrho_{0} G}{v_{2}{ }^{2}} \frac{\partial^{2}}{\partial x_{2}{ }^{2}}\right\} \Gamma_{1}^{*} \\
& -\left\{\left[\left(\lambda+\mu-2 p_{0}\right) \frac{\partial^{2}}{\partial x_{1} \partial x_{2}}+2 i \varrho_{0} \mathcal{S}_{3} \omega\right] \Delta+4 \pi \varrho_{0}^{2} G \frac{\partial^{2}}{\partial x_{1} \partial x_{2}}\right\} \Gamma_{2}^{*} \\
& +\mu \varrho_{0}\left[\square_{2}^{*_{2}} \frac{\partial}{\partial x_{1}}-\frac{2 i \Omega_{3}(1)}{v_{2}{ }^{2}} \frac{\partial}{\partial x_{2}}\right] \Gamma_{3}^{*} \\
& s_{2}^{*}=-\left\{\left[\left(\lambda+\mu-2 p_{0}\right) \frac{\partial^{2}}{\partial x_{1} \partial x_{2}}-2 i \varrho_{0} \Omega_{3} \omega\right] \Delta+4 \pi \varrho_{0}^{2} G \frac{\partial^{2}}{\partial x_{1} \partial x_{2}}\right\} \Gamma_{1}^{*} \\
& +\mu\left\{\left[\square=\frac{v_{1}^{2}}{v_{2}^{2}} \frac{\partial^{2}}{\partial x_{1}^{2}}\right] \Delta+\frac{4 \pi \varrho_{0} G}{v_{2}^{2}} \frac{\partial^{2}}{\partial x_{1}^{2}}\right\} \Gamma_{2}^{*} \\
& +\mu \varrho_{0}\left[\square_{2}^{* 2} \frac{\partial}{\partial x_{2}}+\frac{2 i \Omega_{3} \omega}{v_{2}^{2}} \frac{\partial}{\partial x_{1}}\right] \Gamma_{3}^{*} \text {. } \\
& \Phi_{1}^{*}=-4 \pi \varrho_{0} G \mu\left[\square_{2}^{* 2} \frac{\partial}{\partial x_{2}}+\frac{2 i \Omega_{3}(1)}{v_{2}{ }^{2}} \frac{\partial}{d x_{2}}\right] \Gamma_{1}^{*} \\
& -4 \pi \varrho_{0} G \mu\left[\square_{2}^{*_{2}} \frac{\partial}{\partial x_{2}}-\frac{2 i \Omega_{3} \omega}{v_{2}^{2}} \frac{\partial}{\partial x_{1}}\right] I_{2}^{*} \\
& +\mu\left(\lambda+2 \mu-2 p_{0}\right)\left[\square_{1}^{*_{2}} \square_{2}^{*_{2}}-\frac{4 \Omega_{3^{2}} \omega^{2}}{v_{1}^{2} v_{2}^{2}}\right] \Gamma_{3}^{*}
\end{aligned}
$$

where

$$
\square_{\alpha}^{* 2}=\Delta+\frac{\omega^{2}}{v_{\alpha}^{2}}
$$

The functions $\Gamma_{k}^{*}\left(x_{1}, x_{2}\right)$ satisfy the equations

$$
\begin{gathered}
D^{*} \Gamma_{\alpha}{ }^{*}=-\frac{1}{\mu\left(\lambda+2 \mu-2 p_{0}\right)} F_{\alpha}^{*} \\
D^{*} \Gamma_{3}{ }^{*}=0 .
\end{gathered}
$$


where

$$
D^{*}=\left(\square_{1}^{* 2} \square_{2}^{*_{2}}+\frac{4 \pi \varrho_{0} G}{v_{2}^{2}} \square_{3}^{*_{2}}\right) \Delta,
$$

with

$$
\square_{3}^{\varpi}=\Delta+(1)^{2} / v_{3}^{2}
$$

The operator $D^{+}$can be written in the form:

$$
D^{*}=\Delta\left(\Delta-k_{1}{ }^{2}\right)\left(\Delta-k_{2}{ }^{2}\right),
$$

where $k_{1}{ }^{2}, k_{2}{ }^{2}$ are the roots of the equation

$$
k^{4}+k^{2}\left[\frac{\omega^{2}}{v_{1}^{2}}+\frac{\omega^{2}+4 \pi \underline{Q_{0} G}}{v_{2}{ }^{2}}\right]+\frac{\omega^{2}}{v_{2}^{2}}\left(\frac{\omega^{2}}{v_{1}^{2}}+\frac{4 \pi Q_{0} G\left(\omega^{2}\right.}{v_{3^{2}}^{2}}\right)=0
$$

EFFECT OF CONCENTRATED FORCES.

Let us examine the effect of a borly force acting along the axis $x_{1}$. In this case, we have:

$$
F_{2}=0 \text {, }
$$

and from equations [14], we can take:

$$
\Gamma^{*}=\Gamma_{3}^{*}=0
$$

The solution of the problem is given by:

$$
\begin{aligned}
& s_{1}^{*}=\mu\left\{\left(\Delta, \frac{\omega^{2}}{v_{2}{ }^{2}}+\frac{v_{1}{ }^{2}}{v_{2}{ }^{2}} \frac{\partial^{2}}{\partial x_{2}{ }^{2}}\right) \Delta+\frac{4 \pi \varrho_{0} G}{v_{2}{ }^{2}} \frac{\partial^{2}}{\partial x_{2}{ }^{2}}\right) \Gamma_{1}^{*}, \\
& s_{2}^{*}=-\left\{\left[\left(\lambda+\mu-2 p_{0}\right) \frac{\partial^{2}}{\partial x_{1} \partial x_{2}}-2 i \varrho_{0} \Omega_{3} \omega\right] \Delta+4 \pi \varrho_{0}{ }^{2} G \frac{\partial^{2}}{\partial x_{1} \partial x_{2}}\right\} \Gamma_{1}^{*}, \\
& \Phi_{1}^{*}=-4 \pi \varrho_{0} G \mu\left(\Delta \frac{\partial}{\partial x_{1}}-\frac{\omega^{2}}{v_{2}{ }^{2}} \frac{\partial}{\partial x_{1}}-\frac{2 i \Omega_{3} \omega}{v_{2}{ }^{2}} \frac{\partial}{\partial x_{2}}\right) \Gamma_{1}^{*},
\end{aligned}
$$


where $I_{1}^{*}$ is the solution of the equation:

$$
\Delta\left(\Delta-k_{1}^{2}\right)\left(\Delta-k_{2}^{2}\right) \Gamma_{1}^{*}=-\frac{1}{\mu\left(\lambda+2 \mu-2 p_{0}\right)} F_{1}^{*} \text {. }
$$

We can write the solution of equation [20] in the form:

$$
\Gamma_{1}^{*}=\frac{1}{k_{1}^{2}\left(k_{1}^{2}-k_{2}^{2}\right)} G_{1}-\frac{1}{k_{2}^{2}\left(k_{1}^{2}-k_{2}^{2}\right)} G_{2}+\frac{1}{k_{1}^{2} k_{2}^{2}} G_{3} .
$$

where the functions $G_{i}$ satisfy the equations:

$$
\begin{gathered}
\left(\Delta-k_{\alpha^{2}}\right) G_{\alpha}=-\frac{1}{\mu\left(\lambda+2 \mu-2 p_{0}\right)} F_{1}^{*}, \\
\Delta G_{3}=-\frac{1}{\mu\left(\lambda+2 \mu-2 p_{0}\right)} F_{1}^{*} .
\end{gathered}
$$

Let us consider the concentrated body force

$$
F_{1}^{*}=\delta\left(x_{1}\right) \delta\left(x_{2}\right)
$$

In this case the functions $G_{i}$ are given by:

$$
\begin{aligned}
G_{\alpha} & =\frac{1}{2 \pi \mu\left(\lambda+2 \mu-2 p_{0}\right)} K_{0}\left(k_{\alpha} r\right) \\
G_{3} & =-\frac{1}{2 \pi \mu\left(\lambda+2 \mu-2 p_{0}\right)} \ln \gamma
\end{aligned}
$$

where $K_{0}(z)$ is the modified Bessel function of third kind, and $r^{2}=: x_{1}-+x_{2}{ }^{2}$. Using equations [21] and [24], the solution of equation [20], for concentrated body force, is given by:

$$
\begin{gathered}
\Gamma^{*}=\frac{1}{2 \pi \mu\left(\lambda+2 \mu-2 p_{0}\right)}\left\lfloor\frac{K_{0}\left(k_{1} r\right)}{k_{1}^{2}\left(k_{1}^{2}-k_{2}^{2}\right)}-\frac{K_{0}\left(k_{2} r\right)}{k_{2}^{2}\left(k_{1}^{2}-k_{2}{ }^{2}\right)}\right. \\
\left.-\frac{1}{k_{1}{ }^{2} k_{2}{ }^{2}} \ln r\right]
\end{gathered}
$$

Thus, the solution of the considered problem is given by [19], where $\Gamma_{1}^{*}$ has the expression [25]. In a similar way, we can obtain the solution for a concentrated body force acting along the axis $O x_{2}$. 


\section{ACKNOWLEDGEMENTS.}

I wish to thank Professor A. H. Cook F.R.S. for his kind hospitality in Cambridge, aud Professor M. Caputo for valuable discussions and suggestions.

\section{REFERENCES}

(1) Boscur, E., 1973. - Reciprocity theorem and elastic dislocation theory for an. earth model with an initial static stress field. "J. Geophys. Res.", 78, 8584.

$\left.{ }^{2}\right)$ Boscri, E., 1973. - On the plane strain in a Generalized Theory of Thermoelasticity. Int. "J. Eng. Sci.," 12, 433.

(3) IMArten, F. A., 1972. - Elastic Dislocation. Theory for a Self-Gravitating Elastic Configuration with an Initial Static Stress Field. "Geophys. J. R. Astr. Soc.", 28, 357-383.

(4) IESAN, D., 1968. - On the plane coupled micropolar thermoclasticity. I, "Bull. Acad. Polon. Sci.", Ser. sci. Techn., 16, 379-484.

(5) MorsiL, Gr. C., 1952. - Teoria preliminara a sistemelor de ecuatii cu de. rivate partiale lineare cu coeficienti constanti, Boletinul Stiintific al Acad. R. P. R., Seria A, 4, 319-326.

$\left.{ }^{8}\right)$ NowackI, W., 1964. - Green functions for the thermoelastic medium. II, "Bull. Acad. Polon. Sci.", Ser. sci. Techn., 12, 465-472. 